Department of

Neurology, Institutes of Clinical Medicine

K Ishikawa

$M$ Watanabe

K Yoshizawa

T Fujita

$\mathrm{T}$ Yoshizawa

K Nakamagoe

A Satoh

S Shoji

Department of Pathology, Basic Medical Sciences,

University of Tsukuba, Tsukuba, Japan

T Ogata

Department of Neurology, Mito National Hospital, Mito, Japan

K Yoshizawa

Department of Internal Medicine, Hatsuishi Hospital, Kashiwa, Japan H Iwamoto

Y Komatsuzaki

Department of Internal Medicine, Ibaraki Prefectural

Central Hospital,

Tomobe, Japan

K Harada

Department of Pathology, Tsukuba Medical Center Hospital, Tsukuba, Japan

M Doi

Department of Neurology, Institute of Brain Research, University of Tokyo, Tokyo, Japan

I Kanazawa

Department of Neurology, Tokyo Medical and Dental University, Tokyo, Japan

K Ishikawa

H S Mizusawa

Correspondence to: Dr Hidehiro Mizusawa, Department of Neurology, Tokyo Medical and Dental University, 1-Chome 5-45, Yushima, Bunkyo-ku 113, Tokyo, Japan. Telephone 008135803 5233; fax 0081 0358030134 ;

email h-mizusawa.nuro@

med.tmd.ac.jp

\title{
Clinical, neuropathological, and molecular study in two families with spinocerebellar ataxia type 6 (SCA6)
}

K Ishikawa, M Watanabe, K Yoshizawa, T Fujita, H Iwamoto, T Yoshizawa, K Harada, K Nakamagoe, Y Komatsuzaki, A Satoh, M Doi, T Ogata, I Kanazawa, S Shoji, H Mizusawa

\begin{abstract}
To clarify the clinical, neuropathological, and molecular characteristics of spinocerebellar ataxia type 6 (SCA6), two unrelated Japanese families with SCA6 were studied. A clinical feature of the two families was late onset "pure" cerebellar ataxia. Pathologically, three SCA6 brains consistently showed Purkinje cell dominant cortical cerebellar degeneration. Morphometric analysis showed that loss of the cerebellar granule cells and inferior olivary neurons were very mild compared with the severity of Purkinje cell loss. There was no obvious ubiquitin immunoreactive nuclear inclusions. All affected patients had identical expanded alleles, and the expansion was also homogeneously distributed throughout the brain without mosaicism. The present study showed that SCA6 is characterised by Purkinje cell dominant cortical cerebellar degeneration, highly stable transmission of the CAG repeat expansion, and lack of ubiquitin immunoreactive nuclear inclusions.

(F Neurol Neurosurg Psychiatry 1999;67:86-89)
\end{abstract}

Keywords: SCA6; purkinje cell; CAG repeat; neuropathology

Spinocerebellar ataxia type 6 (SCA6) is an autosomal dominant cerebellar ataxia that is strongly associated with the expansion of trinucleotide (CAG) repeat ${ }^{1}$ in the $\alpha_{1 \mathrm{~A}}$ voltage dependent calcium channel gene (CACNA1A). ${ }^{2}$ We and others ${ }^{3-5}$ further analysed this mutation and found a significant inverse correlation between the number of CAG repeat units in the expanded allele and the age of onset, indicating that the CAG expansion is strongly associated with the pathogenic mechanism of SCA6. Pathology of SCA6 has been also described, ${ }^{67}$ although some variations are seen particularly in the degrees of degeneration of the cerebellar granule cells and inferior olivary neurons. In addition, it is not known whether ubiquitin immunoreactive nuclear inclusion, seen in other diseases associated with CAG repeat expansion, ${ }^{8-11}$ is also present in SCA6.

To define pathological and molecular characteristics as well as clinical features of SCA6, we examined two families, neither of which are the subset of families we described previously. ${ }^{3}$ Here we show that SCA6 has distinct clinical, neuropathological, and molecular features compared with other diseases associated with CAG repeat expansion.

\section{Patients and methods}

CLINICAL INVESTIGATION

Two unrelated Japanese families (UT1 and UT2) were studied. Neuropathological findings and alterations of neurotransmitter markers in one affected member (UT1-IV-5) have been previously described. ${ }^{12}{ }^{13}$ During our subsequent follow up for 12 years, four members further developed ataxia and one patient (UT1-V-7) died, which allowed us to investigate this family more extensively. Clinical examination was performed as described ${ }^{14}$ in all available members $(n=16)$ including at risk family members.

NEUROPATHOLOGICAL INVESTIGATION

Necropsy was performed in three patients: UT1-IV-5 (patient 1; the duration of illness: 28 years), UT1-V-7 (patient 2; 18 years) and UT2-III-1 (patient 3; 14 years). Formalin fixed, paraffin embedded sections were stained with haematoxylin and eosin, Klüver-Barrera, and modified Bielschowsky methods. Immunohistochemistry for ubiquitin (Dako; rabbit polyclonal; dilution 1:400) was also performed with the standard avidin-biotin-peroxidase complex method.

To clarify the degree of neuronal loss, a morphometric analysis was performed in the cerebellar cortex (the culmen, the simple lobule, and the tonsil) and the inferior olivary nucleus (midregion) according to the described methods. ${ }^{12}{ }^{15}$ In brief, sagittal $6 \mu \mathrm{m}$ thick slices of the cerebellum and transverse 10 $\mu \mathrm{m}$ thick slices through the midolivary region were chosen and the Purkinje cells, granule cells, and neurons of the principal inferior olivary nucleus were counted. For each area, the 
Table 1 Morphometric data in the cerebellar cortex and inferior olivary nucleus

\begin{tabular}{|c|c|c|c|c|c|c|c|}
\hline & \multicolumn{3}{|c|}{ Purkinje cells (cells/mm) } & \multicolumn{3}{|c|}{ Granule cells $\left(\times 10^{6}\right.$ cells $\left./ \mathrm{mm}^{3}\right)$} & \multirow[b]{2}{*}{$\begin{array}{l}\text { Inferior olivary neurons } \\
\left(\times 10^{2} \text { cells } / \mathrm{mm}^{3}\right)\end{array}$} \\
\hline & $\begin{array}{l}\text { Superior } \\
\text { vermist }\end{array}$ & $\begin{array}{l}\text { Superior } \\
\text { hemispheret }\end{array}$ & Tonsil & $\begin{array}{l}\text { Superior } \\
\text { vermist }\end{array}$ & $\begin{array}{l}\text { Superior } \\
\text { hemispheret }\end{array}$ & Tonsil & \\
\hline Control $(n=5)$ & 5.64 & 5.88 & 5.93 & 2.24 & 2.21 & 2.16 & 150.83 \\
\hline Patient 1 & $0.42^{\star \star}$ & $4.74^{\star}$ & 4.52 * & $1.67^{\star}$ & $1.86^{\star}$ & 2.12 & 135.47 \\
\hline Patient 2 & $0.37^{\star \star}$ & 5.12 & 5.27 & $1.02^{\star}$ & 2.03 & 2.15 & 129.95 \\
\hline Patient 3 & $0.31^{\star \star}$ & $0.97^{\star \star}$ & $2.53 \star \star$ & $0.77^{\star}$ & $1.50^{\star}$ & 1.84 & $76.26^{\star}$ \\
\hline
\end{tabular}

Patients 1 to 3 correspond to patients UT1-IV-5, UT1-V-7, and UT2-III-1, respectively (see text).

† The superior vermis and superior hemisphere designate the culmen and simple lobule, respectively.

The degree of neuronal loss was evaluated as follows; mild or minimal (no symbols): within $15 \%$ reduction compared with a normal control; moderate ${ }^{\star}$; between 15 and $50 \%$ reduction; severe ${ }^{\star \star}$ : more than $50 \%$ reduction.

Note that the Purkinje cells are consistently and predominantly affected.

numbers of cells were counted in 10 different areas by using an image analyser (Videoplan, Zeiss, Germany), and finally averaged. Five age matched neurologically normal people served as controls.

\section{MOLECULAR ANALYSIS}

After obtaining informed consent, genomic DNA was extracted from peripheral blood lymphocytes in 10 people in UT1. For the three deceased members (patients 1-3), DNA was extracted from the following frozen tissues: the frontal cortex (Brodmann's area 6), occipital cortex (area 17), hippocampus, deep frontal white matter, caudate nucleus, thalamus, pontine base, and cerebellar hemispheric cortex. Except for the deep frontal white matter, CACNA1A is predicted to be expressed abundantly. ${ }^{16}$ The number of CAG repeat units was determined as described. ${ }^{314}$

\section{Results}

CLINICAL FEATURES

The average age of onset was 52.1 (SD 7.3) years $(n=10$; range $37-65$ years). The onset was slightly earlier in offspring than in parents (4.7 (SD 5.9) years), although this difference was not statistically significant. Clinically, our families showed slowly progressive ataxia without any remarkable extracerebellar signs ("pure" cerebellar ataxia). ${ }^{14} 17$ Two members, both within 2 years after onset, showed subtle horizontal gaze nystagmus and mild gait ataxia evident only when performing "tandem gait". None of the patients documented episodic ataxia.

Brain MRI ( $n=7)$ showed restricted atrophy of the cerebellum. Nerve conduction studies, EEG, and somatosensory evoked potentials were all normal $(n=3)$.

\section{NEUROPATHOLOGY}

The brains weighed $1100 \mathrm{~g}$ (patient 1), $1200 \mathrm{~g}$ (patient 2), and $970 \mathrm{~g}$ (patient 3). Macroscopically, all patients showed marked atrophy of the cerebellum particularly in the superior vermis. By contrast, the brainstem and spinal cord were well preserved.

The cardinal histopathological changes were seen in the cerebellar cortex. Loss of Purkinje cells with Bergmann's gliosis was consistenly severe in the superior vermis, whereas it became milder in the inferior vermis and cerebellar hemisphere (table 1). Loss of granule cells was always less severe than the Purkinje cell loss in the corresponding areas. The degree of degeneration was prominent and diffuse in patient 3, whereas both patients from UT1 (patients 1 and 2) had much milder degeneration. There was no apparent neuronal
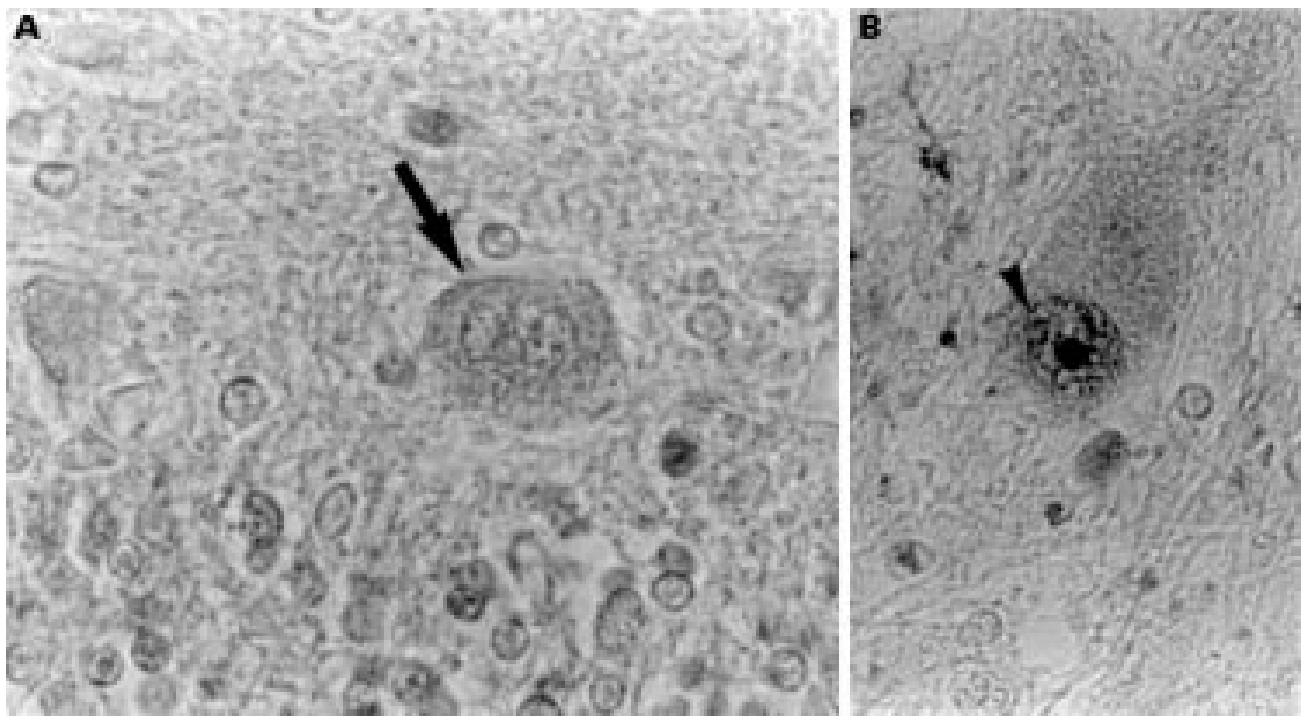

Figure 1 Immunohistochemistry for ubiquitin in the cerebellum with SCA6. (A) There is no obvious ubiquitin immunoreactive nuclear inclusion in a Purkinje cell (arrow) of a patient with SCA6 (immunoperoxidase x250). (B) In a control brain affected with dentatorubral-pallidoluysian atrophy, a ubiquitin immunoreactive inclusion is seen in the centre of the nucleus in a cerebellar dentate neuron. The nucleolus is seen in the periphery of the nucleus (arrowhead; immunoperoxidase, originally $\times 250$ ). 
loss in the dentate nucleus, although mild astrogliosis was seen. In the inferior olivary nucleus, neuronal loss was seen in its dorsomedial region in patient 3 . However, the inferior olivary nucleus was preserved throughout the rostrocaudal extent in patients 1 and 2 .

Other structures were fairly well preserved, except for large cerebral infarcts in patients 1 and 3 , and mild neuronal loss with a few neurofibrillary tangles in the subthalamic nucleus, substantia nigra, and globus pallidus of patient 3. There was no obvious ubiquitin immunoreactive nuclear inclusions in the three brains examined (see fig 1).

MOLECULAR ANALYSIS

All affected members from the two families had expanded alleles with $22 \mathrm{CAG}$ repeat units (fig 2). Normal alleles ranged from 11 to 16 repeats. One asymptomatic member aged 55 years at examination also harboured the same expansion. This member had no neurological signs except for a subtle horizontal gaze nystagmus. In the three SCA6 brains, the number of repeats in the expanded alleles was again identical in all regions examined (fig 2). Both normal and expanded alleles showed single distinct peaks demonstrating no somatic mosaicism.

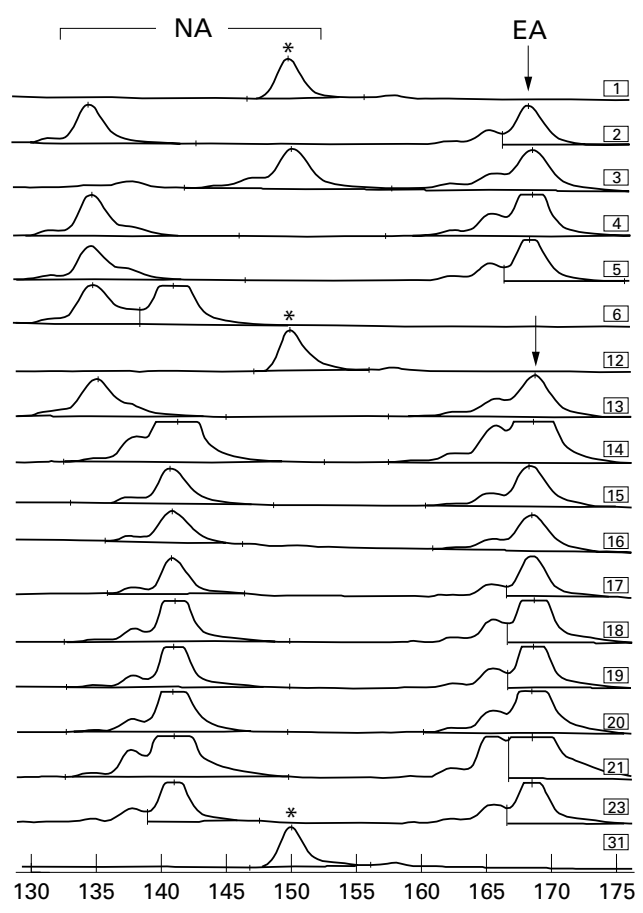

Figure 2 Analysis of the SCA6 CAG repeats. All expanded alleles (EA) show the same 167 base pairs (bp) fragments (corresponding $22 C A G$ repeats; arrows), whereas normal alleles (NA) are seen in variable lengths, between 135 and $150 \mathrm{bp}$ (11 to $16 \mathrm{CAG}$ repeat) range. Note both EA and NA show distinct single peaks. Each lane shows fragments from the following samples: lanes 1 , 12 , and $31=$ external size markers (asterisk $=150 \mathrm{bp}$ ); lanes 2-6=samples from different living members in UT1; lane $13=$ the frontal cortex of patient 1; lane 14=the frontal cortex of patient 2; lane 15=the occipital cortex of patient 2; lane 16=the hippocampus and parahippocampal gyrus of patient 2; lane $17=$ the deep frontal white matter of patient 2 ; lane 18=the caudate nucleus of patient 2 ; lane $19=$ the thalamus of patient 2 ; lane $20=$ the pontine base of patient 2; lane $21=$ the cerebellar cortex of patient 2 ; lane $23=$ the cerebellar cortex of patient 3.

\section{Discussion}

The number of CAG repeats in the expanded alleles in the two families was coincidentally the same 22 repeats, which corresponds to the most common expanded allele in the SCA6 families. ${ }^{13-5}{ }^{18-21}$ Clinical features were summarised as "pure" cerebellar ataxia. Anticipation was not present, as in our previous set of families, ${ }^{3}$ which seems consistent with the fact that all affected members had expansions with identical size. Notably, three patients in a very early stage or in the presymptomatic stage showed only horizontal gaze nystagmus, suggesting that the nystagmus could be the earliest sign of ataxia as well as gait ataxia in SCA6. Although episodic ataxia type 2 (EA-2) and SCA6 clinically overlapped in some families, ${ }^{22}$ none of our affected members documented the episodic nature of ataxia, which fits to the idea that EA-2 and SCA6 have different pathogenic mechanisms. ${ }^{1}$

The present study provided two new pathological aspects of SCA6. Firstly, the present study confirmed that the so called cerebelloolivary atrophy (as in patient 3) and the cortical cerebellar degeneration without obvious olivary involvement (as in patients 1 and 2) could be included under a single entity. ${ }^{23}$ Notably, this pathological variation could not be simply explained by the differences in the length of expansion or the duration of illness. Our finding also indicates that degenerations of granule cells and inferior olivary neurons may occur secondarily to the loss of the Purkinje cells. However, the precise mechanism of degeneration needs to be elucidated, as CACNA1A is also expressed in both the granule cells and olivary neurons. ${ }^{16}$ Secondly, the fact that the ubiquitin immunoreactive nuclear inclusions are absent in SCA6 brains would suggest that the mechanism of neuronal death in SCA6 could be different from those in other CAG repeat diseases. ${ }^{8-11}$ However, recent studies showed that nuclear translocation of the mutant protein is essential, rather than the presence of ubiquitin positive nuclear inclusions, for the pathogenesis of polyglutamine diseases. ${ }^{24}{ }^{25}$ Therefore, it would be important to clarify whether the small CAG repeat expansion in CACNA1A leads to aggregation of the calcium channel protein in the nucleus.

The molecular study showed for the first time that the somatic mosaicism was not seen in various brain regions, where CACNA1A is predicted to be expressed, ${ }^{16}$ or in peripheral blood lymphocytes. Together with the stable transmission within affected family members, this fact would indicate that the transmission of CAG repeats in CACNA1A is very stable, which is by contrast with other "unstable" CAG expansions. ${ }^{26} 27$

In conclusion, SCA6 is characterised by the clinical feature of pure cerebellar ataxia, and predominant Purkinje cell degeneration. Highly stable transmission and lack of ubiquitin immunoreactive nuclear inclusion are features distinct from other diseases associated with CAG repeat expansions. 
We are grateful to the family members described here. This work was supported in part by grants from the Research Committee for Ataxic Diseases, the Ministry of Health and Welfare, Japan (HM), from Brain Science Research, the Ministry of Health and Welfare, Japan (HM), from Scientific Research on Priority areas, the Ministry of Education, Science and Culture, Japan (HM), and from the Japan Society for the Promotion of Science for Young Scientists (KI).

1 Zhuchenko O, Bailey J, Bonnen P, et al. Autosomal dominant cerebellar ataxia (SCA6) associated with smal polyglutamine expansions in the $\alpha 1 \mathrm{~A}$-voltage-dependent calcium channel. Nat Genet 1997;15:62-9.

2 Ophoff RA, Terwindt GM, Vergouwe MN, et al. Familial hemiplegic migraine and episodic ataxia type- 2 are caused by mutations in the Ca2+ channel gene CACNL1A4. Cell 1996;87:543-52.

3 Ishikawa $\mathrm{K}$, Tanaka $\mathrm{H}$, Saito $\mathrm{M}$, et al. Japanese families with autosomal dominant pure cerebellar ataxia map to chromosome 19p13.1-p13.2, and are strongly associated with mild CAG expansions in the spinocerebellar ataxia type 6 gene in chromosome 19p13.1. Am f Hum Genet 1997;61:336-46.

4 Matsuyama Z, Kawakami H, Maruyama H, et al. Molecular features of the CAG repeats of spinocerebellar ataxia 6 features of the CAG repeats of spinoce

5 Reiss O, Schöls L, Böttger H, et al. SCA6 is caused by moderate CAG expansion in the $\alpha 1 \mathrm{~A}$-voltage-dependent calcium channel gene. Hum Mol Genet 1997;6:1289-93.

6 Subramony SH, Fratkin JD, Manyam BV, et al. Dominantly inherited cerebello-olivary atrophy is not due to a mutation at the spinocerebellar ataxia-1, Machado-Joseph disease, or dentato-rubro-pallido-luysian atrophy locus. Mov Disorders 1996;11:174-80

7 Gomez C, Thompson RM, Gammack JT, et al. Spinocerebellar ataxia type 6: gaze-evoked and vertical nystagmus, Purkinje cell degeneration, and variable age of onset. An Neurol 1997;42:933-50.

8 Tellez-Nagel I, Johnson AB, Terry RD. Studies on brain biopsies of patients with Huntington's chorea. I Neuropathol Exp Neurol 1974;33:308-32.

9 Skinner OJ, Koshy BT, Cummings CJ, et al. Ataxin-1 with an expanded glutamine tract alters nuclear matrixan expanded glutamine tract alters nuclear

10 Paulson HL, Perez MK, Trottier Y, et al. Intranuclear inclusions of expanded polyglutamine protein in spinocerebellar ataxia type 3. Neuron 1997;19:333-44.

11 Igarashi S, Koide R, Shimohata T, et al. Suppression of aggregate formation and apoptosis by transglutaminase inhibitors in cells expressing truncated DRPLA protein with an expanded polyglutamine stretch. Nat Genet 1998;18:111-17.

12 Kanazawa I, Kwak S, Sasaki H, et al. Studies on neurotransmitter markers and neuronal cell density in the cerebellar system in olivopontocerebellar atrophy and cortical cerebellar atrophy. 7 Neurol Sci 1985;71:193-208.
13 Mizusawa $\mathrm{H}$, Yoshizawa $\mathrm{K}$, Kanazawa I, et al. A family of cortical cerebellar atrophy (Holmes): a clinical and neuropathological study. Neurol Med 1987;26:257-64

14 Ishikawa K, Mizusawa $\mathrm{H}$, Saito $\mathrm{M}$, et al. Autosomal dominant pure cerebellar ataxia. A clinical and genetic analysis of eight Japanese families. Brain 1996;119:117382.

15 Monagle $\mathrm{RD}$, Brody $\mathrm{H}$. The effect of age upon the main nucleus of the inferior olive in human. 7 Comp Neurol 1974; 155:61-6.

16 Hillman D, Chen S, Aung TT, et al. Localization of P-type calcium channels in the central nervous system. Proc Natl Acad Sci USA 1991;88:7076-80.

17 Harding AE. The clinical features and classification of the ate onset autosomal dominant cerebellar ataxias. A study of 11 families, including descendants of 'the Drew family of Walworth'. Brain 1982;105:1-28.

18 Matsumura R, Futamura N, Fujimoto Y, et al. Spinocerebellar ataxia type 6 . Molecular and clinical features of 35 Japanese patients including one homozygous for the CAG repeat expansion. Neurology 1997;49:1238-43.

19 Stevanin G, Dürr A, David G, et al. Clinical and molecular features of spinocerebellar ataxia type 6. Neurology 1997;49:1243-6.

20 Geschwind DH, Perlman S, Figueroa KP, et al. Spinocerebellar ataxia type 6 . Frequency of the mutation and genotype-phenotype correlations. Neurology 1997;49: 1247-51.

21 Ikeuchi T, Takano H, Koide R, et al. Spinocerebellar ataxia type 6: CAG repeat expansion in $\alpha 1 \mathrm{~A}$ voltage-dependent calcium channel gene and clinical variations in Japanese population. Ann Neurol 1997;42:879-84

22 Jodice C, Mantuano E, Veneziano L, et al. Episodic ataxia type 2 (EA2) and spinocerebellar ataxia type 6 (SCA6) due to CAG repeat expansion in the CACNA1A gene on chromosome 19p. Hum Mol Genet 1997;6:1973-8.

23 Eadie MJ. Cerebello-olivary atrophy (Holmes type). In: Vinken PJ, Bruyn GW, eds. Handbook of clinical neurology. Vinken PJ, Bruyn GW, eds. Handbook of climical neurc

24 Klement IA, Skinner PJ, Kaytor MD, et al. Ataxin-1 nuclear localization and aggregation: role of polyglutamineinduced disease in SCA1 transgenic mice. Cell 1998;95:4153.

25 Saudou F, Finkbeiner S, Devys D, et al. Huntingtin acts in the nucleus to induce apoptosis but death does not correlate with the formation of intranuclear inclusions. Cell 1998;95:55-66.

26 Telenius H, Kremer B, Goldberg YP, et al. Somatic and gonadal mosaicism of Huntington's disease gene CAG repeat in brain and sperm. Nat Genet 1994;6:409-14.

27 Chong SS, McCall AE, Cota J, et al. Gametic and somatic tissue-specific heterogeneity of the expanded SCA1 CAG repeat in spinocerebellar ataxia type 1. Nat Genet 1995;10: $344-9$. 